\title{
Prediction of Aircraft Collision Unsafe Event Based on Hopfield Neural Network Model
}

\author{
XuSheng Gan1,a, Jin-Liang Chen2,b, Hai-Tao Zhao2,c \\ 1 XiJing College, Xi'an, Shaanxi, 710123, China \\ 2 Air Traffic Control and Navigation College, \\ Air Force Engineering University, \\ Xi'an, Shaanxi, 710051, China \\ a ganxusheng123@163.com, b chjl3836@163.com, c zht123@163.com
}

\begin{abstract}
To predict the aircraft collision unsafe event effectively, a feasible modeling and predicting method based on Hopfield neural network is proposed. First, the basic idea of Hopfield neural network is introduced, and then the principle of Hopfield neural network is expatiated. The simulation indicates that the proposed Hopfield neural network model has a good prediction on aircraft collision unsafe event.

Keywords: Aircraft Collision; Influence Factor; Unsafe Event; Hopfield Neural Network

\section{Introduction}

The development of ANN has experienced a tortuous road, so far, a variety of network and its corresponding algorithms have gradually become mature, and have evolved many modified model and algorithm. There exist 40 kinds of neural network models such as Hopfield network, CPN network, BP network, RBF network, and so on [1-4], in which Hopfield network is widely used.

Compare with the prediction problems in other areas, the aircraft collision accident prediction has its particularity. Because the mechanism of aircraft collision accident is often very complex with a certain nonlinear relationship in general, and without showing the model structure, so through the classical regression analysis and time series prediction method, the appropriate model may sometimes be not be established[5]. ANN has strong nonlinear approximation ability and the adaptation ability to the external environment, can be used to deal with the situation. Using the characteristics of ANN to forecast the the aircraft collision accident is more in line with the characteristics of the accident occuring, and can overcome the limitations of the traditional prediction methods that need to construct a structural model with parameters in advance.

The regression prediction based on neural network is that the relevant degree between each influence factor of aircraft collision accident and prediction samples is analyzed by neural network, the future value of each influence is seen as the historical data of time series to mapped to the future data.
\end{abstract}




\section{Basic idea of Hopfield neural network}

Hopfield neural network is an important milepost in the development history of neural network. Hopfield neural network was first proposed in 1982 by American physicist J. J.Hopfield [1][6]. It belongs to the type of feedback neural network. In the feedforward neural network, the relation between the input and output shows just a mapping relationship without considering the time lags influence. But the Hopfield network is different, it uses feedback connections with considering the transmission time delay between the input and output. That is a dynamic process that need to use the differential or differential equations to describe, so the Hopfield network is a kind of feedback systems composed of nonlinear elements, the analysis of its steady state is much more complex than feedforward neural network.

Hopfield uses the idea of energy function theory to form a new calculation method. It illustrates the relationship between neural network and dynamics, and studies the characteristics of neural network based on the nonlinear dynamics method, establishes the criteria on neural network stability, and points out that the information is stored in the connection between each neuron in the network, forming the so-called Hopfield network. Hopfield also compares the feedback network with lsing model in statistical physics in analogy, the upward and downward direction of magnetic rotation are seen as the activation and inhibition states of neurons, the interaction of magnetic rotation is seen as the extruding weights of neuron. In 1984, Hopfield designs and develops the circuit of Hopfleld network model, points out that the neurons can use the operational amplifier to realize, the connection between all neurons can use the electronic circuit to simulate, called continuous Hopfield neural network. By the use of this circuit.

\section{Principle of Hopfield neural network}

Hopfield network is a interconnection network with the feedback, as shown in Fig. 1, where $\mathrm{N}$ is the number of neurons, $\mathrm{V}$ is the input vector of neurons, $\mathrm{U}$ is the output vector, $\mathrm{W}$ is the connection weights between the neurons. The output of each neuron in discrete Hopfield network can only take " 1 " or "- 1 " state, the state available vector $\mathrm{V}$ of each neurons can be expressed as $\mathrm{V}=\{\mathrm{v} 1, \mathrm{v} 2, \ldots, \mathrm{vn}\}$. Each neuron in the network are linked to each other, each neuron transmit its output to other neurons by the connection weights while each neuron receive information from the other neurons. 


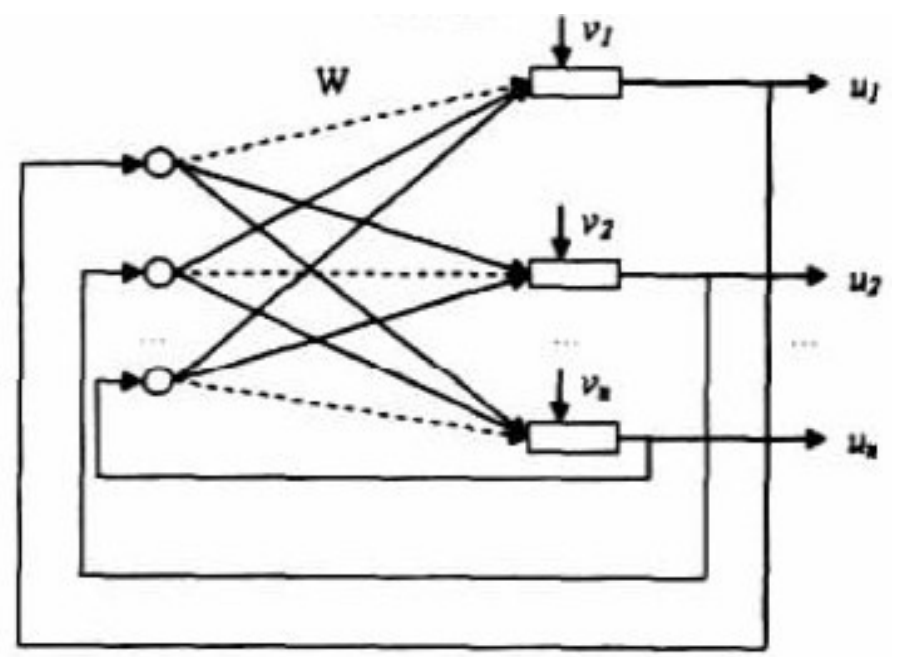

Fig. 1. Hopfield neural network

The stability of the Hopfield network can use the energy function to describe, namely while each state of the network changes, the energy function $\mathrm{E}$ shows the strictly monotone decrease with the changes of network state, so that the stability of Hopfield model has a strict corresponding relationship to the local minimum point that the energy function $\mathrm{E}$ is in the state space.

For Hopfield network with $\mathrm{N}$ neurons, the state of $\mathrm{i}$-th and the $\mathrm{j}$-th neuron node is recorded as vi and vj respectively. $\mathrm{W}$ is the connection weights between neuron i and neuron $\mathrm{j}, \theta$ is the threshold value of neuron i. The energy of the node can be expressed as:

$$
E_{i}=-(
$$

$$
\sum_{j \neq i}^{n} w_{i j} v_{i}-\theta_{i}
$$

(1)

The energy function of entire Hopfield network can be defined as:

$$
E=-\quad \frac{1}{2} \sum_{i=1}^{n} \sum_{j \neq i}^{n} w_{i j} v_{i} v_{j}+\sum_{i=1}^{n} \theta_{i} v_{i}
$$

(2)

Suppose that i-th neuron receives the summation ui(t) of input signal from other $\mathrm{N}-1$ neurons at time $t$. When the output value vi(t+1) of $\mathrm{i}$-th neuron at time $t+1$ is a certain threshold value that the sign function acts on ui(t), the neuron will touch off the excited state. Operation rules of Hopfield network is hereby:

1. Select a neuron randomly in the network;

2. Calculate the summation of the inputs of selected neuron $\mathrm{i}(i \leq N)$ : 
(3)

3. According to ui(t) value, update the state of neuron

$$
\begin{aligned}
& \text { if } u_{i}(t) \geq 0 \\
& \text { then } v_{i}(t+1)=1 \\
& \text { else } v_{i}(t+1)=0
\end{aligned}
$$

4. The state of the neuron $j$ except for neuron $i$ is unchanging

5. Go to step 1, until the network reached a stable.

When Hopfield neural network is used as the learning and memory, the stable state has been given, the suitable weight matrix $\mathrm{W}$ (symmetric matrix) is calculated through network learning, after the learning is completed the association is conducted by the calculaton way. For a given M model, Hopfield network can carry out the learning process according to the Hebb rules.

$$
W_{i j}=\left\{\begin{array}{l}
0, i=j \\
\sum_{k=1}^{M} v_{i}(k) v_{j}(k), i \neq j
\end{array}\right.
$$

After the weight matrix is calculated according to the rules, we can think that the network has make $\mathrm{M}$ mode stored in the contection weight of the network.

\section{Actual example}

The relationship between the aircraft collision unsafe event and the influence factors is very complex and nonlinear, which are often difficult to use the concrete function model to describe. The selection of Hopfield neural network regression model for prediction and analysis is a better choice. Table 1 and Table 2 give the historical statistic data of the aircraft collision unsafe events and the influence factors respectively. We try to use the historical data from January to September to establish the Hopfield network model, and predict the aircraft collision unsafe event from October to November.

Table 1 Statistic data of the influence factors 


\begin{tabular}{|c|c|c|c|c|c|c|c|c|c|c|c|}
\hline Influence factor (times) & Jan. & Feb. & Mar. & Apr. & May. & Jun. & Jul. & Aug. & Sep. & Oct. & Nov. \\
\hline Inappropriate resource management & 1 & 1 & 1 & 0 & 0 & 0 & 0 & 1 & 2 & 1 & 0 \\
\hline Failed to correct problem & 3 & 2 & 4 & 1 & 2 & 1 & 3 & 4 & 2 & 1 & 0 \\
\hline $\begin{array}{c}\text { Inappropriate crew resource } \\
\text { management }\end{array}$ & 8 & 9 & 12 & 7 & 10 & 7 & 6 & 9 & 9 & 10 & 8 \\
\hline Supervisory violations & 3 & 3 & 1 & 2 & 1 & 3 & 1 & 2 & 1 & 3 & 1 \\
\hline Adverse physical environment & 8 & 6 & 7 & 5 & 3 & 3 & 4 & 5 & 6 & 5 & 8 \\
\hline Skill-based errors & 0 & 1 & 1 & 2 & 1 & 1 & 1 & 0 & 0 & 0 & 0 \\
\hline Adverse technological environment & 1 & 0 & 1 & 2 & 1 & 2 & 1 & 1 & 1 & 2 & 1 \\
\hline Adverse organizational process & 2 & 2 & 1 & 1 & 0 & 1 & 1 & 2 & 2 & 0 & 1 \\
\hline Adverse psycho states & 2 & 1 & 2 & 2 & 2 & 0 & 3 & 2 & 0 & 2 & 1 \\
\hline Adverse physiological states & 0 & 1 & 2 & 1 & 0 & 1 & 0 & 1 & 3 & 0 & 2 \\
\hline Decision-making errors & 0 & 2 & 0 & 0 & 2 & 0 & 0 & 0 & 1 & 1 & 1 \\
\hline Flagrant violations & 1 & 0 & 0 & 0 & 0 & 0 & 0 & 0 & 0 & 0 & 1 \\
\hline Physical mental limitations & 0 & 1 & 1 & 0 & 1 & 1 & 0 & 0 & 1 & 1 & 1 \\
\hline Adverse organization climate & 1 & 0 & 2 & 3 & 1 & 2 & 1 & 2 & 3 & 1 & 2 \\
\hline Adverse personal readiness state & 0 & 1 & 1 & 0 & 0 & 1 & 0 & 0 & 1 & 0 & 0 \\
\hline Misperception errors & 0 & 0 & 1 & 1 & 1 & 1 & 1 & 0 & 0 & 1 & 1 \\
\hline Routin violations & 1 & 1 & 2 & 1 & 2 & 0 & 2 & 1 & 1 & 2 & 0 \\
\hline Inadequate supervision & 1 & 0 & 2 & 1 & 0 & 1 & 1 & 1 & 1 & 1 & 0 \\
\hline Inappropriate operation & 1 & 0 & 0 & 0 & 0 & 1 & 0 & 0 & 0 & 1 & 0 \\
\hline
\end{tabular}

Table 2 Statistic data of aircraft collision unsafe events

\begin{tabular}{c|ccccccccccc}
\hline $\begin{array}{c}\text { Aircraft collision } \\
\text { unsafe event }\end{array}$ & Jan. & Feb. & Mar. & Apr. & May. Jun. & Jul. & Aug. & Sep. & Oct. & Nov. \\
\hline Accident & 0 & 0 & 0 & 0 & 0 & 0 & 0 & 0 & 0 & 0 & 0 \\
incident & 1 & 0 & 0 & 0 & 0 & 0 & 1 & 0 & 0 & 1 & 0 \\
Serious error & 4 & 3 & 5 & 3 & 3 & 2 & 2 & 3 & 2 & 4 & 2 \\
General error & 5 & 6 & 8 & 5 & 7 & 6 & 4 & 7 & 8 & 7 & 6 \\
\hline
\end{tabular}

The historical data from January to September in Table 1 are taken as the training input samples, and the historical data from January to September in Table 
2 are taken as the training output samples. The number of hidden layer nodes is 15, which can build the Hopfield neural network model with the structure 19-16-4. The historical data from October to November in Tables 1 and Tables 2 are taken as the test samples. The desired error can be select as 0.00001 .

The prediction results obtained by established Hopfield network regression model are shown in Table 3. From the analysis, the Hopfield neural network model can be used to forecast the aircraft collision unsafe event, and can obtain satisfactory accuracy.

Table 3 Prediction results of aircraft collision unsafe events

\begin{tabular}{c|c|c|c|c}
\hline \multirow{2}{*}{$\begin{array}{c}\text { Aircraft collision } \\
\text { unsafe event }\end{array}$} & \multicolumn{2}{|c|}{ Oct. } & \multicolumn{2}{c}{ Nov. } \\
\cline { 2 - 5 } & Real value & Prediction value & Real value & Prediction value \\
\hline Accident & 0 & $-0.0490(0)$ & 0 & $0.4066(0)$ \\
incident & 1 & $0.7152(1)$ & 0 & $-0.3687(0)$ \\
Serious error & 4 & $3.6400(4)$ & 2 & $1.4374(1)$ \\
General error & 7 & $7.1145(7)$ & 6 & $6.2859(6)$ \\
\hline
\end{tabular}

\section{Conclusions}

For the prediction problem of aircraft collision unsafe event, a new prediction mehod based on Hopfield neural network is proposed. The validation result of actual examples shows that the model established by Hopfield neural network has a good prediction result for aircraft collision unsafe event. Thus, Hopfield neural network can be used to establish the model and predict.

\section{References}

[1] J. J. Hopfield. Neural computation of decisions in optimization problems. Biological Cybernetics, 52, (1987), 141-152

[2] D. E. Rumelhart, J. L. Mcclelland. Parallel distributed processing. Cambridge: MITPress, (1986)

[3] S. Chert, P. M. Crant, C. F. N. Cown. Orthogonal least square algorithm for radial basis function networks. IEEE Transaction on Neural Networks, 2(2), (1991), 302-309

[4] J. L. Elman. Find structure in time. Cognitive Science, 14(2), (1990), 179-211 
[5] H. L. Cui, X. S. Gan, Y. R. Wu, et al. Anti-collision risk assessment method based on relevance vector machine. Applied Mechanics and Materials, 387, (2013), 341-344.

[6] P. Chen, J. F. Guo. Analysis on Hopfield neural network to TSP. Journal of Beijing University of Posts and Telecommunications, 22(2), (1999), 58-61 